\title{
AGN feedback on the ISM of 3C 236
}

\author{
A. Labiano ${ }^{1}$, S. García-Burillo ${ }^{2}$, F. Combes ${ }^{3}$, A. Usero ${ }^{2}$, \\ R. Soria-Ruiz ${ }^{2}$, G. Tremblay ${ }^{4}$, R. Neri ${ }^{5}$, A. Fuente ${ }^{2}$, R. Morganti ${ }^{6,7}$, \\ and T. Oosterloo ${ }^{6,7}$ \\ ${ }^{1}$ Centro de Astrobiología (CSIC-INTA), Madrid, Spain. \\ email: alvaro.labiano@cab.inta-csic.es \\ ${ }^{2}$ Observatorio Astronómico Nacional, 28014, Madrid, Spain. \\ ${ }^{3}$ Observatoire de Paris, LERMA \& CNRS, Paris, France. \\ ${ }^{4}$ European Southern Observatory, Garching bei München, Germany. \\ ${ }^{5}$ Institut de Radioastronomie Millimétrique, St. Martin d'Héres Cedex, France. \\ ${ }^{6}$ Netherlands Foundation for Research in Astronomy, Dwingeloo, The Netherlands. \\ ${ }^{7}$ Kapteyn Astronomical Institute, Groningen, The Netherlands.
}

\begin{abstract}
We have carried out $1 \mathrm{~mm} / 3 \mathrm{~mm}$ continuum and ${ }^{12} \mathrm{CO}(2-1)$ line high resolution observations to identify the footprints of AGN feedback on 3C 236. The CO emission comes from a spatially resolved disk characterized by a regular rotating pattern. Within the limits imposed by the sensitivity and velocity coverage of our data, we do not detect any outflow signatures in the cold molecular gas. Re-inspection of optical and IR spectra, shows the presence of a previously unknown ionized gas outflow. The star-formation efficiency in 3C 236, is consistent with the value measured in normal galaxies, which follow the canonical Kennicutt-Schmidt law. This result, confirmed to hold in other young radio sources examined in this work, is in stark contrast with the factor of 10-50 lower SFE that has been claimed to characterize evolved powerful radio galaxies. The recent reactivation of the AGN in $3 \mathrm{C} 236$ is a likely explanation for the early evolutionary status of its molecular disk.
\end{abstract}

Keywords. galaxies: individual (3C 236) — galaxies: ISM — galaxies: kinematics and dynamics - galaxies: active - ISM: jets and outflows

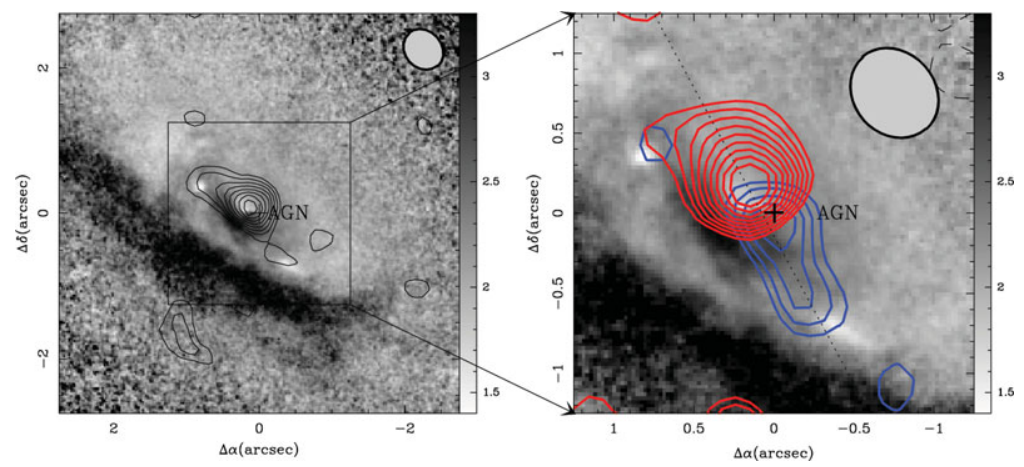

Figure 1. Left: V-H color map (gray scale in STmag magnitude system) of 3C 236, with the integrated ${ }^{12} \mathrm{CO}(2-1)$ emission overlaid. The contour levels are $3 \sigma$, to $6.5 \sigma$ in steps of $0.5 \sigma$, with $\sigma=0.21 \mathrm{Jy} \mathrm{km} \mathrm{s}^{-1}$. Right: Zoom into the central 3". Contours represent the ${ }^{12} \mathrm{CO}(2-1)$ line maps integrated in two channels: blue $\left(v_{\mathrm{sys}}^{\mathrm{CO}}-330 \mathrm{~km} \mathrm{~s}^{-1}<v<v_{\mathrm{sys}}^{\mathrm{CO}}\right)$ and $\operatorname{red}\left(v_{\mathrm{sys}}^{\mathrm{CO}}<v<v_{\mathrm{sys}}^{\mathrm{CO}}+485 \mathrm{~km}\right.$ $\mathrm{s}^{-1}$; color version of the figure is available online). The contour levels for the blue channel are $0.39 \mathrm{Jy}^{\mathrm{beam}}{ }^{-1}$ to $0.91 \mathrm{Jy}$ beam ${ }^{-1}$ in steps of $0.065 \mathrm{Jy} \mathrm{km} \mathrm{s}^{-1}\left(\sigma=0.12 \mathrm{Jy} \mathrm{km} \mathrm{s}^{-1}\right)$. The contour levels for the red channel are $0.39 \mathrm{Jy}$ beam $^{-1}$ to $0.59 \mathrm{Jy}^{\mathrm{beam}}{ }^{-1}$ in steps of $0.065 \mathrm{Jy} \mathrm{km} \mathrm{s}^{-1}$ $\left(\sigma=0.14 \mathrm{Jy} \mathrm{km} \mathrm{s}{ }^{-1}\right)$. The dashed line shows the major axis of the molecular gas disk.

Full details about this work can be found in Labiano et al. AछA, in press. 